MATEC Web of Conferences 34, 04007 (2015)

DOI: $10.1051 /$ matecconf/ 20153404007

(C) Owned by the authors, published by EDP Sciences, 2015

\title{
Intelligent Control Framework for the Feeding System in the Biomass Power Plant
}

\author{
Jin Sun ${ }^{1, \text { a }}$, Xinglong Zhu ${ }^{1}$, Xiaowei Cao ${ }^{1}$, Jing Ding ${ }^{1}$ \\ ${ }^{1}$ Yangzhou University in Yangzhou, Jiangsu, China
}

\begin{abstract}
This paper proposes an intelligent control framework for biomass drying process with flue gases based on FLC (fuzzy logic controller) and CAN (Controller Area Network) bus. In the operation of a biomass drying process, in order to get the biomass with the set-point low moisture content dried by waste high temperature flue gases, it is necessary to intelligent control for the biomass flow rate. Use of an experiment with varied materials at different initial moisture contents enables acquisition of the biomass flow rates as initial setting values. Set the error between actual straw moisture content and set-point, and rate of change of error as two inputs. the biomass flow rate can be acquired by the fuzzy logic computing as the output. Since the length of dryer is more than twenty meters, the integration by the CAN bus can ensure real-time reliable data acquisition and processing. The control framework for biomass drying process can be applied to a variety of biomass, such as, cotton stalk, corn stalk, rice straw, wheat straw, sugar cane. It has strong potential for practical applications because of its advantages on intelligent providing the set-point low moisture content of biomass feedstock for power generation equipment.
\end{abstract}

\section{Introduction}

Since the bio-oil , as a sort of renewable energy, is easy to transport and store, the interest in producing liquid fuels from biomass is increasing. Before thermo-chemical treatments, such as gasification or combustion, the biomass can be dried by dryers as the input feedstock for a subsequent power plant through. In practical processes, the reactor which often operates at high pressure and/or high temperature causes additional challenges in establishing reliable feeding system. In feeding system, such as the biomass flow rate, biomass moisture content, surface roughness, the variety of biomass, and so on can all affect the reliable and successive feeding speed. In addition, an required uniform and continuous flow of the feedstocks need be provided in biomass utilization processes. Since how to feed the biomass feedstocks in the required uniform and continuous mode is a critical problem, control method may be the key of the feeding system. To address the problem, some new methods have been developed. In 2012, Yan $\mathrm{Ru}[1]$ used single-chip microcomputer and wireless communication to achieve the goal of real time monitoring and automatic feeding to the drug machines. Guangfeng Chen proposed[2] a CANbus and stepper motor based yarn feeding control system for tufting carpet machine. In 2013, Lanjun Li[3] put forward a control system based on Advanced RISC Machines, touch panel and PLC to control the operation of feeding conveyer in automatic tomato selection

\footnotetext{
a Corresponding author: sunjin1001@,126.com
}

assembly line. Zhangtao Yin[4] designed the monitor control system using LOGO as the core control in the tomato sorting assembly line. In 2014, Xijuan Wang[5] selected PLC as control unit to control the corresponding food supply line's movement according to the signal from the selected dishes. However, little information has been focused on how to intelligent control to get the required uniform and continuous flow of the feedstock. In this paper, an intelligent control framework based on PLC,FLC and CAN bus was devised. This paper is organized as follows. Section 1 presents the instruction of biomass feeding system and the literature review of the methods of getting a reliable and successive feeding. Section 2 presents the components of the biomass feeding system, and the intelligent control framework for the feeding system is proposed. The technique of PLC and FLC can provide the required uniform and continuous flow of the feedstocks and intelligently meet the final moisture content. The technique of CAN bus can ensure real-time reliable data acquisition and processing. Section 3 presents an example of applications of the proposed approach and the testing result of the changes of water content. Section 4 provides the summary and some extensions of our study.

\section{Methods}




\subsection{Feeding system}

The feeding system can be seen as shown in Figure 1. The wet materials were conveyed from the hopper to the chain conveyors by bucket elevator. Then they entered into the unpacking $\& \&$ loosing machine through the charge of the distribution trolleys. In the unpacking \&\& loosing machine, the sealed straw packages were quickly cut for unpacked and loosed. The loose straw feedstocks were fed into the belt dryer through the belt conveyor. In the belt dryer, the wet materials were be dried by waste high temperature flue gases from high moisture content to a set-point low moisture content. Then the materials are discharged to the material warehouse through the screw conveyor. In addition, the unpacked wood chips firstly were conveyed by $1 \#$ belt conveyor, and discharged to $2 \#$ belt conveyor from the plow unloader, and then they were fed into the belt dryer through the belt conveyor. $3 \#$ belt conveyor was a backup conveyor.

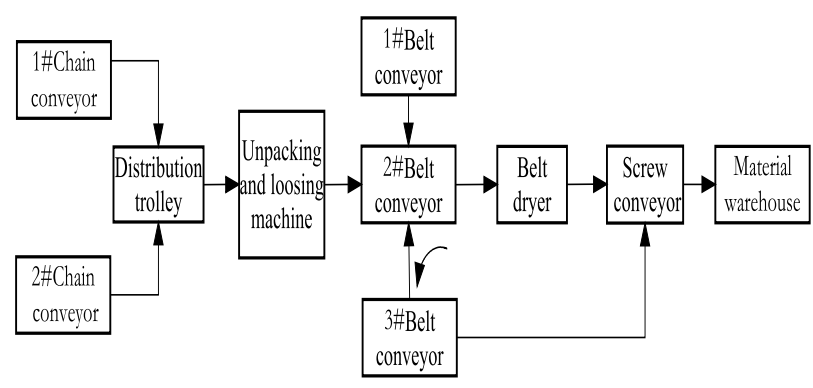

Figure 1. The schematic diagram of feeding system

\subsection{Intelligent control framework for the feeding system}

In this section, an intelligent control framework for the feeding system was described. The part of 2.2.1 and 2.2.2 proposed the PLC control of chain conveyor \&\& distribution trolley, unpacking\&\&loosing machine, respectively. The part of 2.2.3 introduced fuzzy logic control for belt dryer and D part set CAN bus for reliable data acquisition and processing.

\subsubsection{PLC control of chain conveyor \&\& distribution trolley}

As shown in Figure 2 was the PLC control workflow of chain conveyor \&\& distribution trolley. Wherein the conversion conditions were as follows: $\mathrm{X} 0$ : the chain conveyor received the feeding signal from distribution trolley; X1: the chain conveyor received the material bags from the hopper; X2: the chain conveyor had no material; $\mathrm{X} 3$ : the distribution trolley received the feeding signal from unpacking \&\& loosing machine; $\mathrm{X} 4$ : the distribution trolley received the material bag; X5: the distribution trolley unloaded the material bag. When in automatic operation mode, after received feeding signal from distribution trolley and material bags from the hopper, chain conveyor began to start up and run. When received feeding signal from unpacking \&\& loosing machine, distribution trolley started up. After received material bags from chain conveyor, distribution trolley began to run. After the material bags were unloaded, the distribution trolley stopped. If the whole process was controlled by manually stop, the chain conveyor $\& \&$ the distribution trolley stopped.

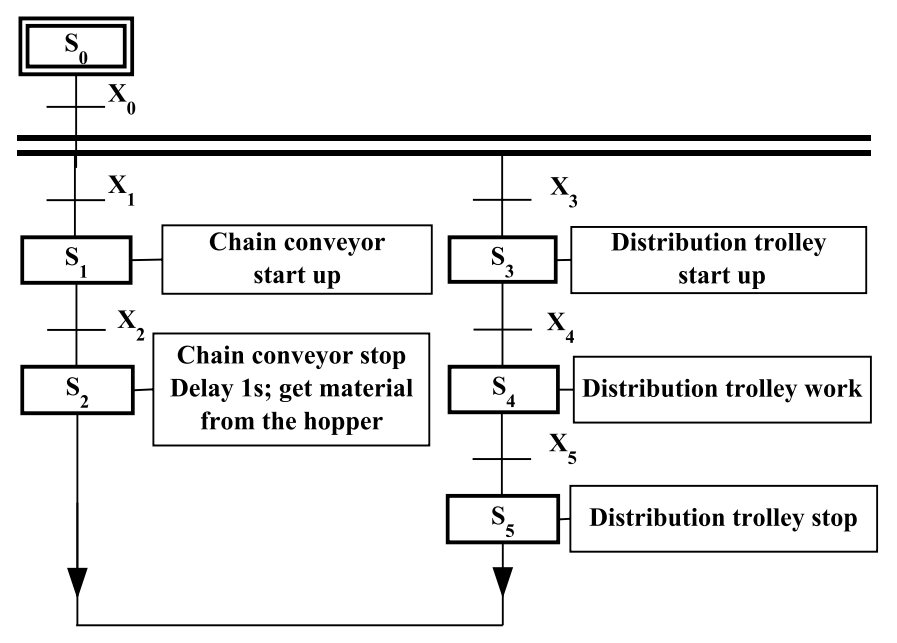

Figure 2. PLC control workflow of chain conveyor $\& \&$ distribution trolley

\subsubsection{PLC control of unpacking \&\& loosing machine}

As shown in Figure 3, the unpacking \&\& loosing machine was produced by Jiangsu chaoyang hydraulic mechanism group Co.,LTD in China. After being put on the carry chain, the raw material bags moved ahead. When they reached at the unpacking machine, two cutting blades quickly cut down the rope to quick unpack the material bags. Then the unpacked materials reached at the loosing machine. First along the guided roller, then a pair of transition roller held the materials, the knife on the roller cut the materials into short-length pieces. At last the shorten and loose materials were delivered into the belt conveyor.

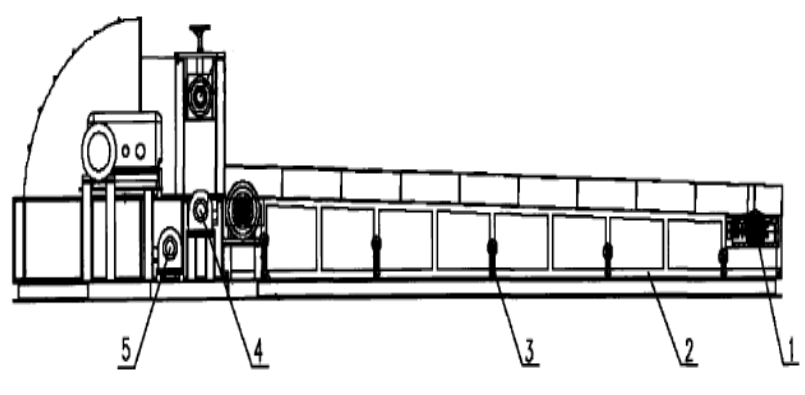

1Tail shaft; 2 frame;3 carry chain;4 unpacking machine;5 loosing machine

Figure 3. The schematic diagram of unpacking $\& \&$ loosing machine

As shown in Figure 4 was the PLC control workflow of unpacking \&\& loosing machine, wherein the conversion conditions were as follows: $\mathrm{X} 1$ : the carry chain received the feeding signal from belt conveyor; X2: the carry chain received the material bag from distribution trolley; X3: the carry chain received the stop signal from 
unpacking machine; $\mathrm{X} 4$ : the unpacking machine received the signal that the carry chain received the material bag; $\mathrm{X} 5$ : the unpacking machine received the material bag; X6: the unpacking machine received the stop signal from loosing machine; X7: the loosing machine received the signal that the unpacking machine received the material bag; X8: the loosing machine received the material bag;X9: the loosing machine unloaded the material bag. When in automatic operation mode, after the feeding signal was received from belt conveyor, the carry chain began to start up. As soon as the material bags from the unpacking machine were received, the loosing machine began to run. After the material bags were unloaded, the loosing machine stopped, and then the carry chain and the unpacking machine also stopped one after another.

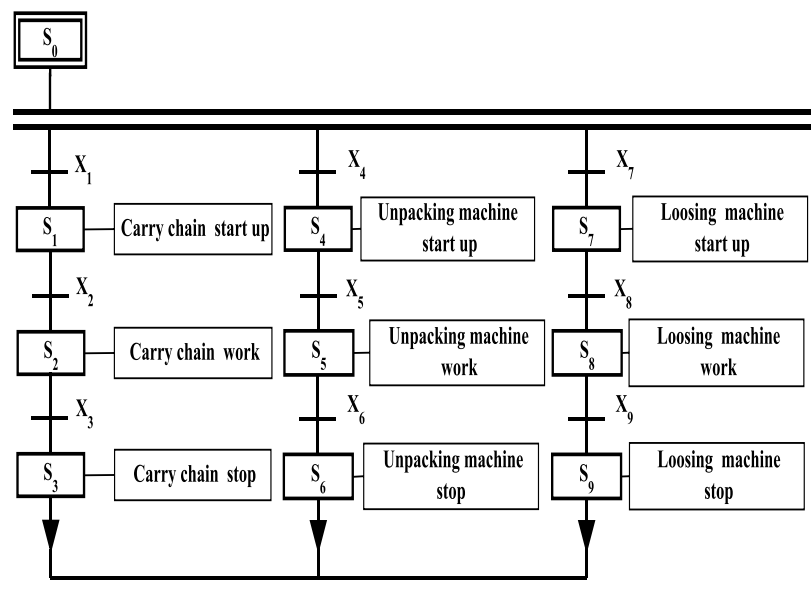

Figure 4. PLC control workflow of unpacking \&\& loosing machine

\subsubsection{Fuzzy logic control for belt dryer}

In the belt dryer, the wet feed stocks need to be dried from nearly $60 \%$ to a set-point low moisture content, such as $15 \%$. An fuzzy logic control framework was proposed for straw drying process, which can adjust the feedstock flow rate to meet the final moisture content. The fuzzy control was designed with mamdani method and center of gravidity defuzzifier.

Firstly, the humidity error and weight error were system inputs and output variable was straw flow rate.

i) Humidity error $H_{e}$

$$
\begin{gathered}
H_{e}=\left(\Delta H_{s e t}-\Delta H_{a c t}\right) \times 100 \% ; \\
\Delta H_{s e t}=H_{p r i}-H_{s e t} ; \\
\Delta H_{a c t}=H_{p r i}-H_{a c t}
\end{gathered}
$$

Where $H_{s e t}, H_{p r i}$ and $H_{a c t}$ were humidity set-point, primary humidity and actual final humidity value after dying, respectively. We defined five sets: Negative, Negative small, Zero, Positive small, Positive, where Negative means $H_{\text {act }}$ is bigger than $H_{\text {set }}$.

ii) Weight error $W_{e}$

$$
W_{e}=\frac{W_{p r i}-W_{a c t}}{W_{p r i}} \times 100 \%
$$

Where $W_{p r i}$ was the initial weight and $W_{a c t}$ was the final weight. Weight variable was a good parameter to indicate the amount product drying rate. The weight variable was defined with five sets: Negative, Negative small, Zero, Positive small, Positive, where Zero was a desired weight requested by the user and reaching to this weight meant the end of operation; Negative means the drying rate is too slow and Positive means the drying rate is too quick.

iii) The output variable was straw flow rate. The seven sets Very slow, Slow, Not so slow, Normal, Not so fast, fast, very fast were defined for straw flow rate.

Secondly, the membership functions were defined depending on the need for every input and output. The membership functions of inputs were defined as gaussmf type and the membership functions of output was defined as trimf type which are good indicators of their sets.

Thirdly, the fuzzy rules were written based on input's and output's membership functions. With correct rules, we can expect an appropriate control system. The general form of rules in this article is as follows: IF $<$ weight error is ...> AND $<$ humidity error is ... $>$ THEN $<$ Straw flow rate is ...>. According to membership functions, 25 rules were written.

At last, the surface was achieved for the desirability of system's behavior. As shown in Figure 5, there is a coordinate system composed of three coordinates: the straw flow rate, the humidity error and the weight error. The coordinates of the straw flow rate range from 0 to1; The coordinates of the weight error range from -0.2 to 0.2 ; The coordinates of the humidity error range from -0.1 to 0.1 . The surface of straw flow rate was plotted based on humidity and weight error. The smoother the surface was, the better the performance of the system was. From Figure 5,we can find that the surface of straw flow rate is smooth and the transition is slow, that means, the performance of the system is as good as be accepted.

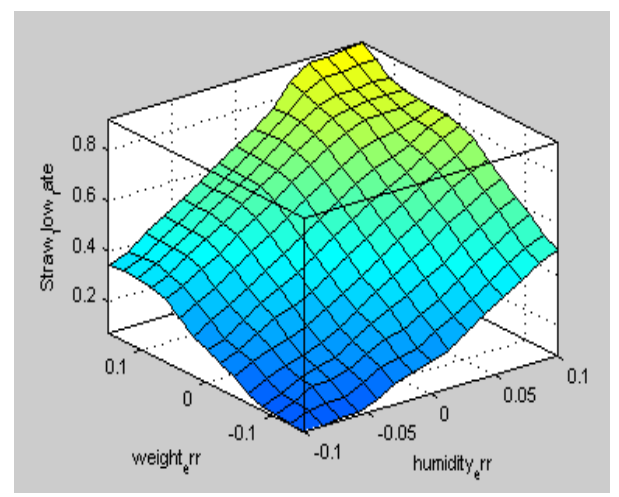

Figure 5. The surface of straw flow rate based on humidity and weight error

\subsubsection{CAN bus setting for reliable data acquisition and processing}

CAN bus is a fieldbus suitable for general industrial applications since CAN bus offers many advantages such 
as high reliability, short delay times, error handling and multi-master ability, and so on. CANopen[6] is an application layer communication protocol based on CAN bus. CANopen device is often consist of communication protocol, object dictionary and application programs. The communication protocol defines four categories of communication objects: Service Data Object, Process Data Object, Network management and special function objects. "Service Data Object" is used to visit the object dictionary, and to configure the control parameters; "Process Data Object" is served as the transmission of real-time data; "Network management" is used for the monitoring and management of master node for the passive node and the special functional objects can communicate and network management of the above three categories of objects.

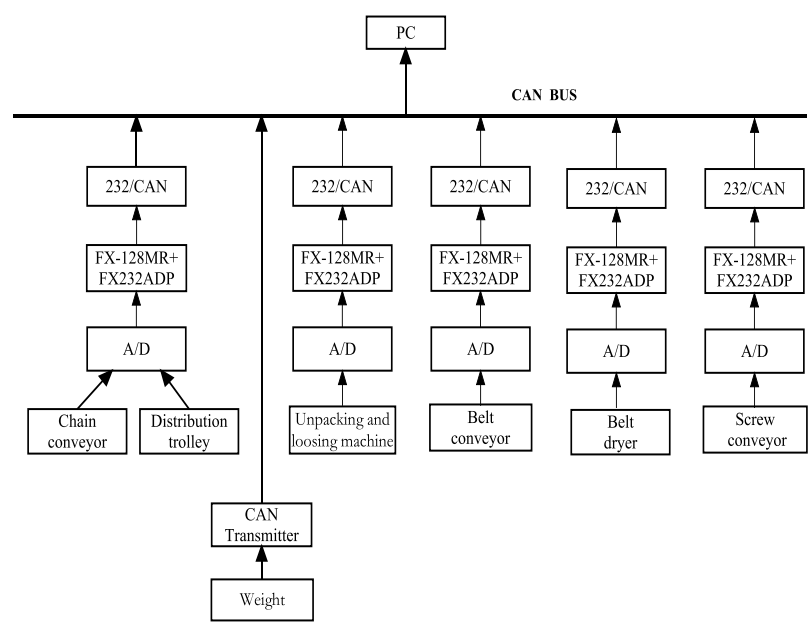

Figure 6. CAN bus setting for reliable data acquisition and processing

As shown in Figure 6 was the CAN bus setting for reliable data acquisition and processing. There were five modules which are "chain conveyor and distribution trolley", "unpacking and loosing machine", "belt conveyor", "belt dryer" , "screw conveyor". They were controlled by PLC of FX-128MR. RS232 can merely realize point to point communication, and $R S 485 / 422$ can only enable communication within 32 nodes. When the distance was beyond twenty metres, their antiinterference ability was relatively weak. In this paper, we used the industrial PC with built-in PC-CAN interface card to build one CANopen network. The 232 / CAN gateway and FX232ADP allowed each PLC to have the interface connecting with CANopen bus. The CANopen Communication can greatly simplify the implementation of distributed applications in CAN based systems[7]. We can visit "application objects" of the device (such as input / output device parameters and functions and network variables, etc.) from the object dictionary entry. The entry of the object dictionary was often determined by an index and sub-index uniquely. The PC can be used to configure for SDO devices, and to exchange high-speedily for data between PDO and the CANopen devices. Each PLC can set a separate device ID number, which length is 11 or 29 , as the address of the PLC unit. In such a network, the number of PLC serials can be unlimited theoretically.
Any PLC can initiate the data communications. By means of the "virtual serial port" software, CANopen network[8] can build up to up to 2047 standard serial communication ports, which can connect up to up to 2047 serial network. It was shown that CANopen provides flexible real-time data transfer mechanisms that were able to meet timecritical constraints. Furthermore, CANopen can make a good solution for distributed control environments, especially for such a feeding system in the biomass power plant.

\section{System Application and Testing}

The production test site was shown in Figure 7.

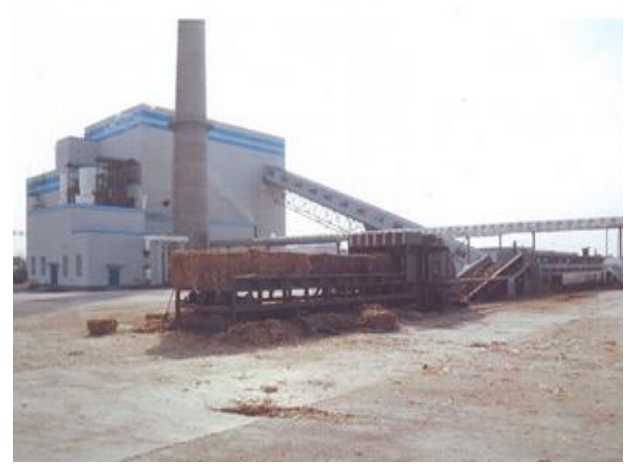

Figure 7. The production test site and test result

Use of an experiment with varied materials at different initial moisture contents enables acquisition of the biomass flow rates as initial setting values. Test material was corn straw.Set the error between actual straw moisture content and set-point, and rate of change of error as two inputs. the biomass flow rate can be acquired by the fuzzy logic computing as the output. The temperature of flue gases are set as $130{ }^{\circ} \mathrm{C}$. The initial moisture content were set as $40 \%$ and the expected moisture content was $15 \%$.The final result illustrated as shown in Figure 8. that the intelligent control framework based on PLC,FLC and CAN bus can satisfy the requirement, since it can work in an uniform and continuous feeding rate. And finally provide the feedstocks with the set-point low moisture content.

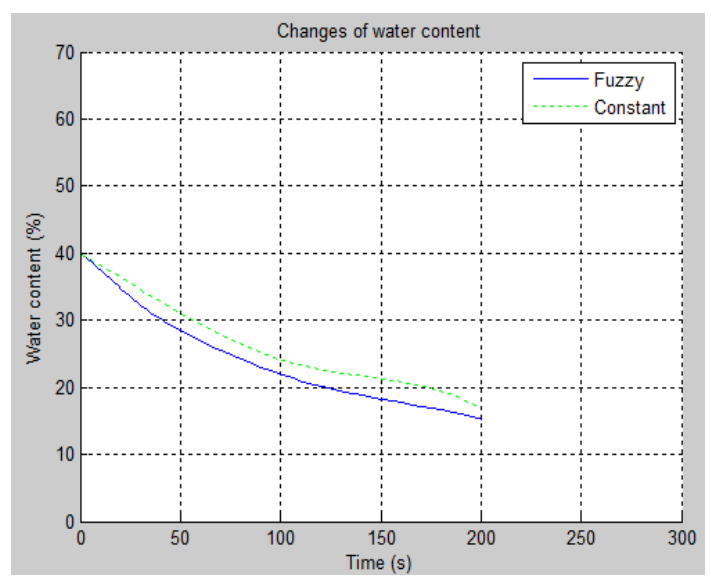

Figure 8. The changes of water content 


\section{Conclusions}

Successful feeding of biomass can provide considerable benefits for subsequent power plant. Biomass feeding process has a nonlinear process and multivariable model achieving which necessitates hard work. In order to get the required uniform and continuous flow rate and the biomass with the set-point low moisture content dried by waste high temperature flue gases, this paper proposed an intelligent control framework for biomass feeding process based on PLC,FLC and CAN bus. The technique of PLC and FLC can provide the required uniform and continuous flow of the feedstocks and adjust the biomass flow rate intelligently to meet the final moisture content. And the technique of CAN bus can ensure real-time reliable data acquisition and processing. The control framework had strong potential for practical applications because of providing feedstock with required uniform and continuous feeding rate and the set-point low moisture content for power generation equipment.

\section{Acknowledgments}

This work was supported by National Natural Science Foundation of China (No. 51475409), Jiangsu Province Basic Research Program Natural Science Foundation (No. BK20141277), China Postdoctoral Science Foundation Funded Project (No. 2013M541736), Jiangsu Planned Projects for Postdoctoral Research Funds (No. 1302179C), and Yangzhou city - Yangzhou University of Science and Technology Cooperation Program Funds (No. 2012038-12).

\section{References}

1. $\mathrm{Ru}$ Y. Research on Feeding Vehicle Control System for Drug Packaging. Journal of Convergence Information Technology, 2012, 7(22): 644-651.

2. Chen G F, Zhai L L, ZOU K, et al. Design of CANBus Based Yarn Feeding Control System for Carpet Tufting Machine. Advanced Materials Research, 2012, 459:275-279.

3. Li L, Gao S, Luo Q, et al. Design and implement of ARM-based tobacco feeding control system; proceedings of the Control and Decision Conference (CCDC), 2013 25th Chinese, F, 2013. IEEE: 510-513.

4. Yin Z T, Zhang R Y, Kan Z, et al. Control System Design of Feeding Conveyer in Tomato Automatic Sorting Assembly Line Based on the LOGO!. Applied Mechanics and Materials, 2013, 278:1570-1576.

5. Wang X J, Zhang J J, Feng J X, et al. The Design and Research of Automatic Control Feeding System; proceedings of the Applied Mechanics and Materials, F, 2014 Trans Tech Publ: 1035-1039.

6. L Pez - L Pez F, Gomis - Bellmunt O, Teixid - Casas $\mathrm{M}$, et al. A novel educational platform to teach CANopen field bus. Computer Applications in Engineering Education, 2011, 19(2): 377-384.

7. Yu G, Zhou C, Huang S. A Protocol for Automatic Node-ID Binding in CANopen Networks. Journal of Communications, 2012, 7(10): 765-773.
8. Luo Y, Jiao X, Ji W, et al. Control Network Communication for Pipeline Welding Based on CANopen. Procedia Engineering, 2011, 15(1): 49944999. 